\title{
Coarse-grained interaction potentials for polyaromatic hydrocarbons
}

O. A. von LilienfeldDenis Andrienko

Citation: The Journal of Chemical Physics 124, 054307 (2006); doi: 10.1063/1.2162543

View online: http://dx.doi.org/10.1063/1.2162543

View Table of Contents: http://aip.scitation.org/toc/jcp/124/5

Published by the American Institute of Physics

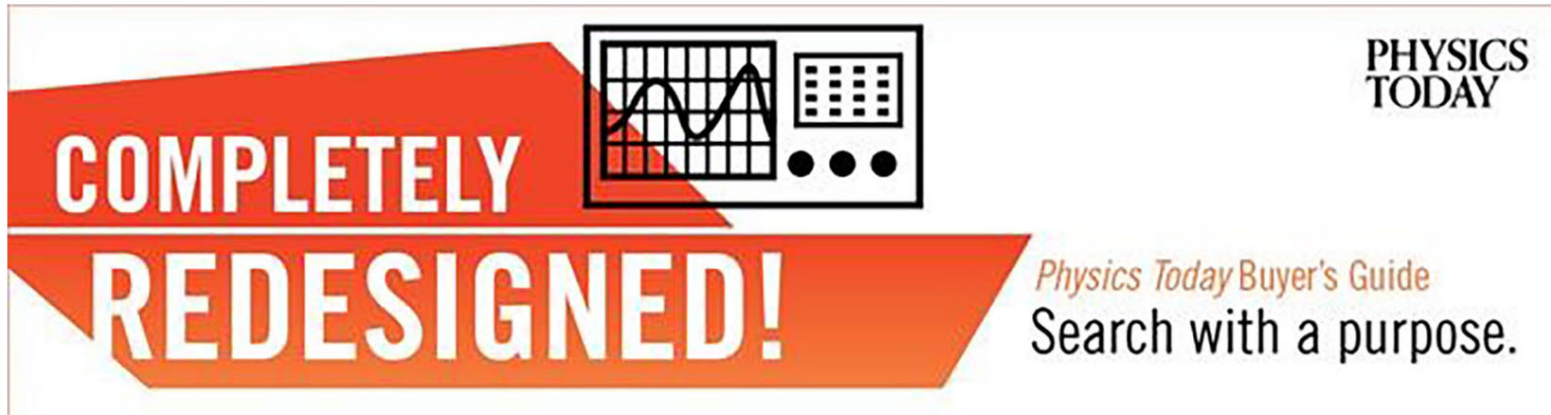




\title{
Coarse-grained interaction potentials for polyaromatic hydrocarbons
}

\author{
O. A. von Lilienfeld ${ }^{\text {a) }}$ \\ Department of Chemistry, New York University, New York, New York 10003 and Institute for Pure and \\ Applied Mathematics, University of California Los Angeles, 460 Portola Plaza, Los Angeles, California \\ 90095-7121 \\ Denis Andrienko ${ }^{\text {b) }}$ \\ Institute for Pure and Applied Mathematics, University of California Los Angeles, 460 Portola Plaza, Los \\ Angeles, California 90095-7121 and Max-Planck-Institut fur Polymerforschung, Ackermannweg 10, \\ 55128 Mainz, Germany
}

(Received 16 November 2005; accepted 7 December 2005; published online 1 February 2006)

\begin{abstract}
Using Kohn-Sham (KS) density-functional theory, we have studied the interaction between various polyaromatic hydrocarbon molecules. The systems range from monocyclic benzene up to hexabenzocoronene (hbc). For several conventional exchange-correlation functionals total potential-energy curves of interaction of the $\pi-\pi$ stacking hbc dimer are reported. It is found that all pure local density or generalized gradient approximated functionals yield qualitatively incorrect predictions regarding structure and interaction. Inclusion of a nonlocal, atom-centered correction to the KS Hamiltonian enables quantitative predictions. The computed potential-energy surfaces of interaction yield parameters for a coarse-grained potential, which can be employed to study discotic liquid-crystalline mesophases of derived polyaromatic macromolecules. (C) 2006 American Institute of Physics. [DOI: 10.1063/1.2162543]
\end{abstract}

\section{INTRODUCTION}

Discotic thermotropic liquid crystals can be formed by flat molecules with a central aromatic core and several aliphatic chains attached at the edges. ${ }^{1,2}$ The size and the shape of the cores can be varied, as well as the length and the structure of the side chains, which allows the control of functional properties of these mesophases. ${ }^{3-5}$ Liquid-crystalline properties such as fluidity are of help to process these compounds and even to develop self-healing materials.

The self-organization of the aromatic cores into $\pi-\pi$ electron-bonded stacks surrounded by saturated hydrocarbons allows one-dimensional charge transport along the columns. ${ }^{6,7}$ Unfortunately, the spatial arrangement of stacks is not perfect, i.e., the columns can be misaligned, tilted, or form various types of topological defects. In addition, the local alignment of molecules in columns can vary for different compounds. This considerably affects the intermolecular overlap of the $\pi$ orbitals and thereby the efficiency of the charge transport in a single column. As a consequence, the details of the morphology of the conducting film are crucial. $^{8-10}$ An accurate in silico prediction of meshophase properties prior to the actual synthesis of the compounds could account for a considerable gain in efficiency on the route towards the design of macromolecular photodevices. ${ }^{11}$

An accurate understanding of the constituting molecules and their intermolecular interactions is mandatory for controling the local alignment of the disks or the global arrangement of the columns in the mesophase. Depending on the

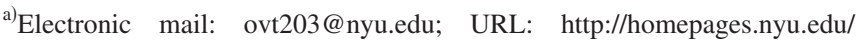
$\sim$ ovt203/

${ }^{b}$ Electronic mail: denis.andrienko@mpip-mainz.mpg.de; URL: http:// www.mpip-mainz.mpg.de/
}

chosen length and time scales different methods can be considered. In principle, at the quantum chemistry level, one can study electronic, inter- and intramolecular adsorption and adhesion processes, ${ }^{12}$ and even compound design ${ }^{13}$ without empiricism. Still at an atomistic resolution-but using empirical molecular force fields-nanometer and nanosecond simulations can yield local properties, such as order parameters or molecular arrangements. ${ }^{14-17}$ In an even more extended time and length scale limits ( $\mu \mathrm{m}$ and $\mu \mathrm{s})$, coarsegrained simulations allow to describe the morphology of bulk material, global arrangement of macromolecular objects such as columns or generic phase diagrams, and defects. ${ }^{18-24}$

To our knowledge, discotic materials have been studied only very little and if so with idealized model potentials. The reason being that an $a b$ initio treatment of the dispersion forces is computationally very demanding. Molecular dynamics (MD) is able to treat larger systems; however, the details of the electronic structure, which are crucial for an understanding of electron transport, are by construction not included in MD. Moreover, it requires empirical parameters for the atom-atom interactions, and defects and the mesophase morphology can only be studied at even more coarse levels. Consequently, multiscale methodologies ${ }^{25-31}$ seem to represent the most adequate and tractable description of these systems.

The aim of this work is to make first steps towards multiscale modeling of discotic mesophases of polyaromatic hydrocarbons. Namely, coarse-grained potentials for the interaction between representative polyaromatic molecules in a face-to-face geometry are obtained from first principles. They can be applied to the study of macroscopic properties of these materials or their chemically derived structures. 


\section{COMPUTATIONAL DETAILS}

The intermolecular attraction between polyaromatic systems is partly attributed to London-dispersion forces. These forces result from the correlated fluctuation of nonoverlapping electron densities of molecular fragments. ${ }^{32}$ Their prediction from first principles has remained a long-standing challenge because of the very high accuracy required to describe electron correlation effects. Explicitly correlated wave-function methods such as coupled-cluster, configuration interaction, or quantum Monte Carlo allow for an accurate treatment of these forces but are computationally prohibitively expensive for all but the smallest polyaromatic hydrocarbons, such as for instance the benzene dimer. ${ }^{33}$ Kohn-Sham density-functional theory (KS-DFT), on the other hand, would be an exact electronic structure method if the true exchange-correlation (xc) term in the KS potential was known. Unfortunately, this is not the case and for all practical purposes approximations have to be made. While some of the pure xc functionals fortuitously but inconsistently predict binding for London-dispersion complexes, it is not yet generally possible to describe van der Waals (vdW) interactions correctly within DFT using the local-density approximation (LDA), the generalized gradient approximation (GGA) or even the-on average more accurate-hybrid exchange-correlation functionals. ${ }^{34-39}$

Considering the ubiquitous nature of these intermolecular forces and their importance for self-assembly, much effort is being devoted to design superior xc potentials which can account correctly for all intermolecular interactions. The use of nonlocal correlations by electron density partitioning ${ }^{40,41}$ can efficiently remedy this deficiency but implies an a priori assignment of molecular fragments. A "van der Waals" functional as proposed in Refs. 42-46, and based on response theory becomes rapidly intractable, such as the schemes described in Refs. 47 and 48. As a consequence, empirical $a$ posteriori pairwise atom-atom based correction terms ${ }^{37,49}$ to the energy are in wide spread use for practical applications. The required parameters and damping functions for the correct repulsive behavior can be obtained from experiments or from various theoretical approaches including timedependent DFT. ${ }^{50-53}$ However, these $r^{-6}$-dependent corrections to the energy and ionic forces need artificial damping functions to allow for the correct repulsive behavior, and more importantly leave the electronic structure uncorrected.

In this study, London-dispersion forces are computed from an improved electronic structure calculation which exploits a recently presented semiempirical dispersion calibrated atom-centered (DCACP) correction $\hat{v}_{i}^{\text {disp }}$ to a given Hamiltonian. ${ }^{54}$ Specifically, it can be seen as a nonlocal extension of a given, local for LDA or GGA, xc potential,

$$
\hat{v}_{\mathrm{xc}}^{\text {extended }}(\mathbf{r})=\hat{v}_{\mathrm{xc}}(\mathbf{r})+\sum_{i} \hat{v}_{i}^{\text {disp }}\left(\mathbf{r}, \mathbf{r}^{\prime},\{\lambda\}\right),
$$

where index $i$ enumerates all atoms. As generally suggested in Ref. 55, the atom-type-dependent parameters $\{\lambda\}$ require preliminary calibration for an improved electronic structure fulfilling additional requirements, such as exerting a Londondispersion force on the ions. Specifically, the BLYP-DCACP

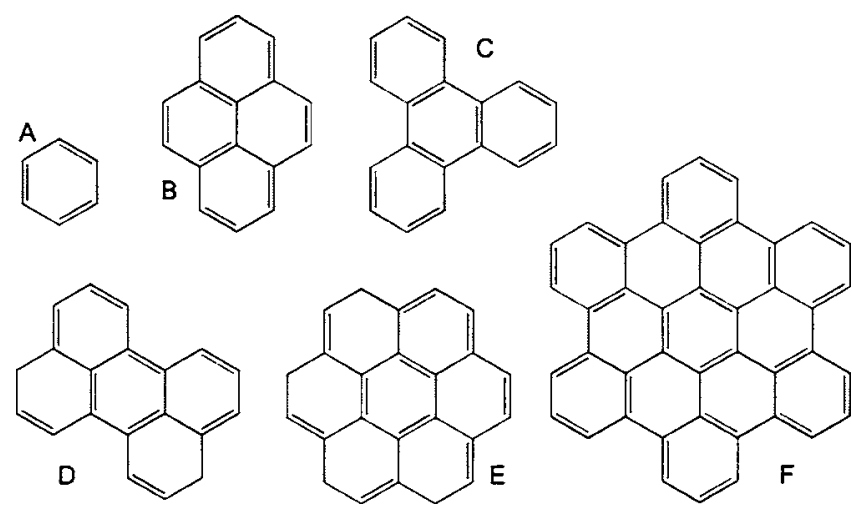

FIG. 1. Sketches of the polycyclic aromatic hydrocarbons benzene (A), pyrene $(\mathrm{B})$, triphenylene $(\mathrm{C})$, perylene $(\mathrm{D})$, coronene $(\mathrm{E})$, and hexabenzocoronene (hbc) (F). All hydrogens are omitted for the sake of clarity. Bonds are represented by edges, while carbon atoms are represented by vertices.

for carbon as it has been introduced, calibrated to the benzene dimer, and assessed in Refs. 54, 56, and 57 is employed. Generalization of this correction to other xc functionals than BLYP has already been carried out. ${ }^{58}$

All DFT calculations have been carried out using the plane-wave basis-set electronic structure program CPMD $3.92,{ }^{59}$ the xc-functionals BLYP $,{ }^{60-62} \mathrm{BP},{ }^{60,63} \mathrm{PBE},{ }^{64}$ LDA (using the Perdew and Zunger fit ${ }^{65}$ to the data of Ceperley and Alder $^{66}$ ), Goedecker pseudopotentials from Refs. 67-69, and a plane-wave cutoff of $100 \mathrm{Ry}$. The isolated system module in CPMD has been employed together with the Poisson solver of Tuckerman and Martyna. ${ }^{70}$ The box size is sufficiently converged at $19 \times 19 \times 20 \AA^{3}$ for the largest system (hbc) and has been kept fixed for all molecules and all distances. Carbon-type DCACPs (Ref. 54) have been used only as a correction to the BLYP functional, no correction has been employed for hydrogen atoms. For the calibration of the DCACP's in Ref. 54, the Møller-Plesset second-order perturbation theory energy of interaction of the benzene dimer was used as a reference. Since the plane-wave basis is independent of atomic positions, no basis-set superposition errors occur. Relative energies have been computed for identical box sizes and cutoffs. For all geometry optimizations the residual tolerance for ionic forces has been set to 0.0005 a.u. For all calculations of energies of interaction, the monomer geometries have been optimized with the given xc functional. Thereafter, for the calculation of the interaction curves, the intramolecular geometries of the top-on-top moieties have been hold fixed and only the intermolecular distance has been varied.

Several aromatic disks with symmetric cores have been selected. Sketches of their chemical composition are shown in Fig. 1. Namely, complexes of benzene (A), pyrene (B), triphenylene $(C)$, perylene $(D)$, coronene $(E)$, and hexabenzocoronene (hbc) (F) have been studied, which all fulfill Hückel's $(4 N+2)$ rule.

\section{RESULTS AND DISCUSSION}

\section{A. Different density functionals}

First, several xc functionals have been assessed by evaluating the potential energies of interaction, 


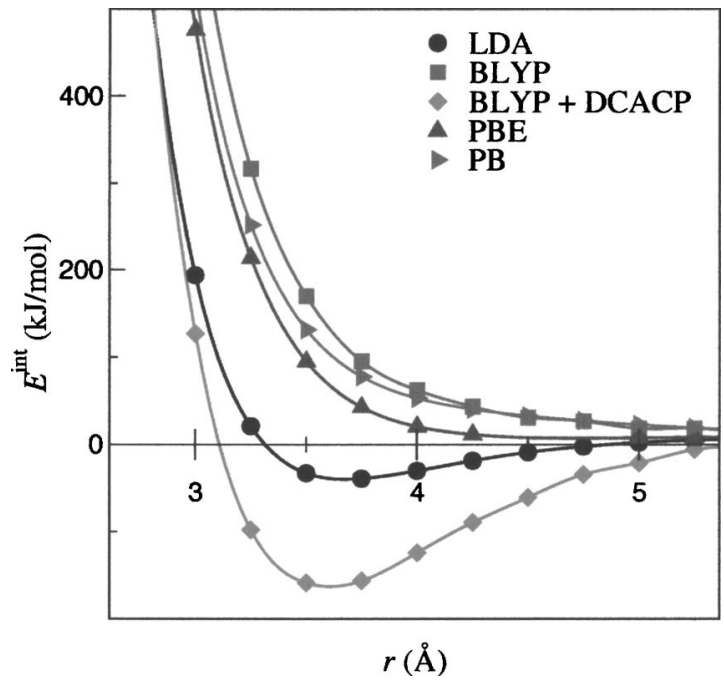

FIG. 2. Total potential energy of interaction for hexabenzocoronene (see structure F, Fig. 1) as a function of intermolecular distance for the LDA, BLYP, PBE, BP, and BLYP+DCACP xc functionals.

$$
E^{\text {int }}=E^{\text {dimer }}-2 E^{\text {monomer }},
$$

for hexabenzocoronene using LDA, BLYP, PBE, and BP. The results are shown in Fig. 2. As expected, LDA exhibits some fortuitous binding for graphite-type structures, however, when using the more sophisticated GGA functionals, a completely repulsive behavior is obtained. It is found that the $\mathrm{PBE}$ gives the least repulsive interaction, followed by $\mathrm{BP}$, and BLYP, suggesting that Becke's exchange potential is too repulsive for the investigated systems. Upon inclusion of the DCACP extension into the BLYP-DFT Hamiltonian, the interaction energy curve is in reasonable agreement with what can be expected from experimental results, available for coronene. $^{71}$

\section{B. Interaction energy profiles}

The interaction energy profiles $E^{\text {int }}(r)$ have been calculated for all systems in the face-to-face geometry using the BLYP-DCACP KS Hamiltonian. The results are presented in Fig. 3. The calculated profiles are interpolated with cubic splines. The equilibrium separations $r_{\mathrm{eq}}$ and the minima of the interaction energy $E_{\mathrm{eq}}^{\text {int }}$ are determined from the interpolated curves and are reported in Table I, together with the value for two isolated graphene sheets from Ref. 54.

When increasing the disk size of the systems, the energy of interaction per carbon atom increases; correspondingly, the equilibrium separation decreases. The remaining difference of the largest system with respect to graphene is most probably due to the static multipole of the saturating hydrogen atoms, which is known to represent up to $7 \%$ of the interaction energy in the case of benzene. ${ }^{72}$ The convergence of the interaction parameters with the system's size can be exploited for extrapolations to even larger structures, such as supernaphtalene or supertriphenylene, ${ }^{73}$ for which the importance of symmetry and hydrogen atoms can be expected to decrease, due to the even smaller ratio between molecular perimeter and surface.

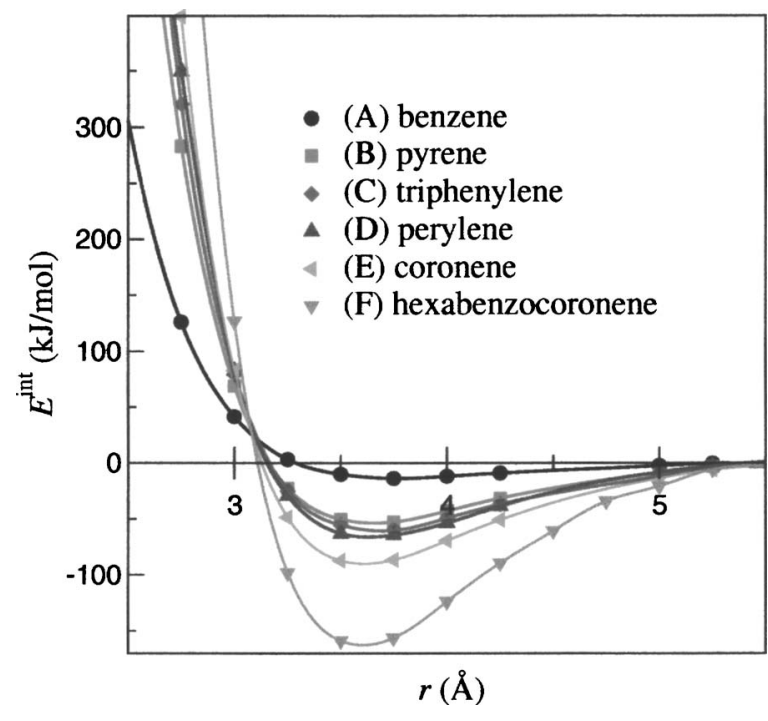

FIG. 3. Energies of interaction vs molecule-molecule separation for all studied aromatic systems. Calculations are performed using the BLYP + DCACP functional.

To obtain a coarse-grained interaction potential we have normalized the energies by the interaction energy at the equilibrium distance, $E_{\text {eq }}^{\mathrm{int}}$, and have scaled the molecule-molecule separation with the equilibrium distance $r_{\text {eq }}$. After scaling all DFT profiles superimpose on a single curve, as illustrated in Fig. 4. This suggests that the main contribution to the nonbonded interactions of the atoms in the dimer is due to an (additive) pairwise carbon-carbon interaction.

We have fitted the master curve with three frequently used potentials: Lennard-Jones (LJ), Morse, and Buckingham. All the potentials have been constrained to have the minimum of the energy, $u_{\mathrm{eq}}=-1$, at the dimensionless separation, $x_{\text {eq }}=1$. Under this constraint, the Lennard-Jones potential is parameter free, while the Morse and Buckingham potentials have each a single fitting parameter,

$$
\begin{aligned}
& u_{\mathrm{LJ}}(x)=\left[\frac{1}{x^{12}}-\frac{2}{x^{6}}\right], \\
& u_{\mathrm{Morse}(x)}=\left[1-e^{-\alpha(x-1)}\right]^{2}-1,
\end{aligned}
$$

TABLE I. Minimum of the interaction energy $E_{\text {eq }}^{\text {int }}$ together with the equilibrium distance $r_{\text {eq }}$ for all the systems. Graph corresponds to the calculated prediction for two graphene sheets in vacuum which compares well to experiment.

\begin{tabular}{lrcc}
\hline \hline \multicolumn{1}{c}{ System } & $N$ & $-E_{\text {eq }}^{\text {int }}(\mathrm{kJ} / \mathrm{mol})$ & $r_{\mathrm{eq}}(\AA)$ \\
\hline (A) benzene & 6 & 13.8 & 3.77 \\
(B) pyrene & 16 & 53.7 & 3.67 \\
(C) triphenylene & 18 & 60.6 & 3.70 \\
(D) perylene & 20 & 66.3 & 3.63 \\
(E) coronene & 24 & 90.1 & 3.60 \\
(F) hexabenzocoronene & 42 & 162.7 & 3.60 \\
Graph $^{\text {a }}$ & 42 & 140.7 & 3.30 \\
\hline \hline
\end{tabular}

${ }^{\mathrm{a} F r o m ~ R e f . ~} 54$. 


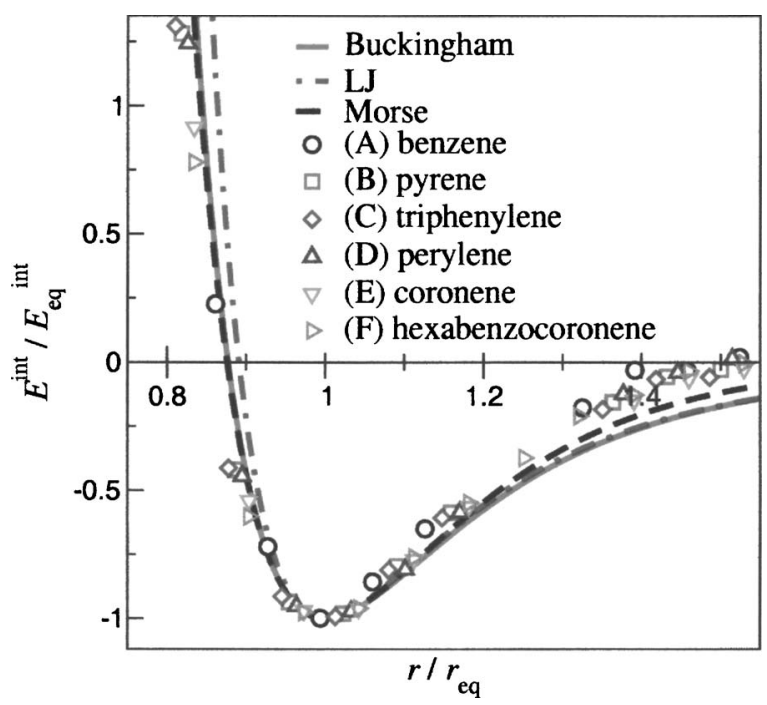

FIG. 4. Interaction energies in units of $E_{\mathrm{eq}}^{\mathrm{int}}$ vs the separation scaled by $r_{\text {eq. }}$. The DCACP-BLYP-DFT results (symbols) for all systems fall on a single master curve. Solid lines represent fits corresponding to different coarsegrained potentials, Lennard-Jones, Morse, and Buckingham.

$$
u_{\text {Buckingham }}=\frac{1}{\beta-6}\left[6 e^{-\beta(x-1)}-\frac{\beta}{x^{6}}\right] .
$$

Here, $x=r / r_{\text {eq. }}$. The interval $x=[0.9,1.4]$ has been used to determine the fitting parameters $\alpha=5.6$ and $\beta=12.3$.

The dimensional potential is obtained by multiplying $u(x)$ with the absolute value of $E_{\mathrm{eq}}^{\mathrm{int}}$ and using $x=r / r_{\mathrm{eq}}$ as the argument. The corresponding values are given in Table I. For instance, the Lennard-Jones potential for benzene would have a potential energy of interaction of $13.8 \times u_{\mathrm{LJ}}(r / 3.77)$, in $\mathrm{kJ} / \mathrm{mol}$, and $r$ in $\AA$.

All the three potentials fit very well to the DFT calculations. The Morse potential reproduces best the repulsive and the attractive part of the potential. The fact that all interaction potentials fall on a single master curve implies that the performance of the fits remains constant for all investigated supermolecular systems.

\section{Coarse-grained potentials}

\section{United atoms model}

Equations (3), can, in principle, be used directly for the parametrization of interaction potentials treating the whole molecule as one interacting point, such as the Gay-Berne potential. Here, however, we will be interested in a more accurate representation. We start with a united atom model, in which all hydrogen atoms are embedded into the carbons they saturate. All considered molecules are assumed to be rigid, i.e., no stretching, bending, or torsional energy is included. Since, already for benzene, the electrostatic contribution represents up to $7 \%$ of the total intermolecular energy, ${ }^{72}$ we have neglected this contribution for the parametrization of the coarse-grained model.

For atomistic two-body potentials the interaction of two molecules is a sum of the corresponding pair interactions of all atoms. If the effective dispersion-repulsion interaction between two atoms (or united atoms) $i$ and $j$ of different molecules is represented by a Lennard-Jones 6-12 potential,

$$
U^{i j}\left(r_{i j}\right)=4 \varepsilon_{i j}\left[\left(\frac{\sigma_{i j}}{r_{i j}}\right)^{12}-\left(\frac{\sigma_{i j}}{r_{i j}}\right)^{6}\right],
$$

then the molecule-molecule interaction is the sum over all pair interactions of the atoms belonging to different molecules,

$$
U(1,2)=\sum_{i \in 1, j \in 2} U^{i j}\left(r_{i j}\right) .
$$

In what follows we have assumed that all inner and edge carbons have the same parameters, $\varepsilon$ and $\sigma$, and optimized these parameters to reproduce the desired molecule-molecule interaction. For the fit of the DFT data to Eq. (5), we have considered only the region close to the equilibrium separation $r_{\text {eq. }}$. This limitation is due to the fact that the employed DCACP correction to the DFT functional ${ }^{54}$ was calibrated to reproduce only this equilibrium region, and does not explicitly include the typical dissociative $r^{-6}$ behavior.

The resulting parameters of the fit are summarized in Table II. The DFT data points, together with the corresponding fitting curves are shown in Fig. 5(a). Again, the LennardJones potential does not reproduce perfectly well the attractive tail but, as explained before, the position of the minimum is more important for our purposes.

We can also compare some of the obtained parameters to values of existing force fields. For instance, a number of united atom force fields are available for benzene. ${ }^{74-79}$ Depending on the employed parametrization, the literature values are $\sigma \in[3.25-3.57] \AA$ and $\varepsilon \in[0.4-0.75] \mathrm{kJ} / \mathrm{mol}$, i.e., the values of this study fall into the range of parameters predicted from thermodynamic properties of benzene.

For the investigated systems, no abundant experimental data, particularly concerning dimer interactions, are avail-

TABLE II. Lennard-Jones parameters for united atom $(\varepsilon, \sigma)$ and benzene-bead $\left(\varepsilon_{B}, \sigma_{B}\right)$ parametrizations of all the studied systems. The cutoff $r_{\text {cut }}=15 \AA$ has been used to evaluate the potential in Eq. (5). Energies $\omega, \varepsilon_{B}$ are in $\mathrm{kJ} / \mathrm{mol} ; \sigma$ and $\sigma_{B}$ are in $\AA$. $N$ is the number of carbons and $N_{B}$ is the number of benzere beads per molecule.

\begin{tabular}{lrrrrrr}
\hline \hline \multicolumn{1}{c}{ System } & $N$ & $\varepsilon$ & $\sigma$ & $N_{B}$ & $\varepsilon_{B}$ & $\sigma_{B}$ \\
\hline (A) benzene & 6 & 0.466 & 3.556 & 1 & 13.802 & 3.358 \\
(B) pyrene & 16 & 0.408 & 3.541 & 4 & 5.019 & 3.428 \\
(C) triphenylene & 18 & 0.407 & 3.569 & 4 & 6.963 & 3.417 \\
(D) perylene & 20 & 0.383 & 3.512 & 5 & 4.984 & 3.390 \\
(E) coronene & 24 & 0.393 & 3.498 & 7 & 3.536 & 3.413 \\
(F) hbc & 42 & 0.352 & 3.518 & 13 & 2.873 & 3.459 \\
\hline \hline
\end{tabular}




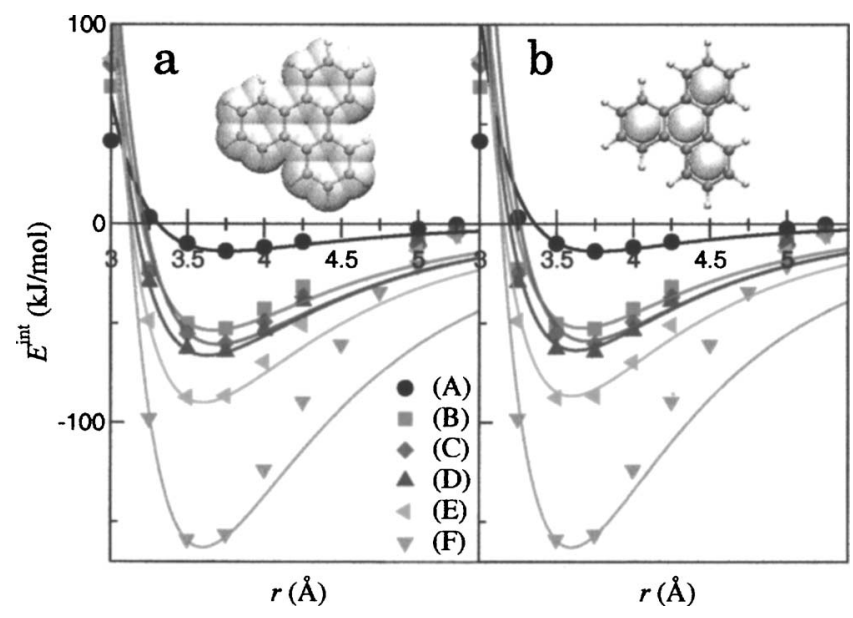

FIG. 5. DFT-data and corresponding fitting curves for united atoms (a) and benzene-bead (b) representations. The insets illustrate the representations for triphenylene $(\mathrm{C})$.

able. However, adsorption energies of benzene or coronene on basal planes of graphite were measured, ${ }^{71} \approx 50$ and $\approx 120 \mathrm{~kJ} / \mathrm{mol}$, respectively. While we find no quantitative agreement with the results for benzene, the agreement for coronene is better. It is plausible that due to the interaction of a molecule with the bulk of graphite, the adsorption energy on graphite is larger than the interaction energy between two isolated benzene molecules.

\section{Benzene-bead representation}

In coarse-grained simulations, one frequently encounters the approximation that fragments building up larger systems are rigid, i.e., their internal stretching, bending, or torsional energy contributions are neglected. Exploiting this assumption, a computationally more efficient coarse-grained representation can be proposed in which each benzene is represented as an interacting point located at its center of mass. This reduces the number of degrees of freedom considerably. Assuming, likewise, a Lennard-Jones type of interaction, the above presented procedure to fit to the DFT data has been applied to obtain the corresponding parameters for benzene beads. The results and fitting curves are displayed in Table II and Fig. 5(b), respectively. There is no significant difference in the interaction profiles upon use of the united atom or the benzene-bead representation. However, for the latter the size of the beads is relatively small, i.e., only as large as the internal diameter of a benzene unit. This implies that only mesophases in which molecules experience interactions via a face-to-face arrangement can be studied. Another limitation is that the rotational profile of the interaction energy, e.g., due to azimuthal rotation of a moiety, has not been included in the parametrizations. Consequently, an accurate prediction of the helix structure, often observed in columnar mesophases, cannot be expected.

\section{CONCLUSIONS}

The DFT KS potentials using LDA, BP, PBE, or BLYP approximations to the xc potential fail to correctly predict an attractive interaction between polyaromatic hydrocarbon molecules. The BLYP-DCACP extension of Ref. 54 has been successfully applied to compute interaction energies for hexabenzocoronene, coronene, perylene, triphenylene, pyrene, and benzene, without any computational overhead or necessity of a priori assignments of fragments. By scaling the obtained data with equilibrium energy and distance values, a single function has been found to describe all molecule-molecule interactions independent of the number of atoms.

The DFT results have been used to parametrize a united atom representation, taking each carbon as an interacting site. Additionally, another coarse-grained representation has been proposed and parametrized in which each benzene unit represents one Lennard-Jones bead.

The obtained interaction potentials will be of use for future studies of columnar phases of corresponding compounds or their derivatives within atomistic moleculardynamics simulations or more coarse representations.

\section{ACKNOWLEDGMENTS}

Both authors would like to thank the organizers of the workshop Bridging Time and Length Scales in Materials Science and Bio-Physics which was held at the Institute for Pure and Applied Mathematics at the University of California, Los Angeles and which inspired this study. Furthermore, the authors thank L. Delle Site and K. Kremer for helpful discussions and support. One of the authors (O.A.v.L.) is grateful for support from the SNF (Grant No. PBEL2-11024).

${ }^{1}$ S. Chandrasekhar and G. S. Ranganath, Rep. Prog. Phys. 53, 57 (1990).

${ }^{2}$ R. J. Bushby and O. R. Lozman, Curr. Opin. Colloid Interface Sci. 7, 343 (2002).

${ }^{3}$ J. D. Brand, C. Kubel, S. Ito, and K. Mullen, Chem. Mater. 12, 1638 (2000).

${ }^{4}$ S. Kumar, Liq. Cryst. 31, 1037 (2004).

${ }^{5}$ M. Muller, C. Kubel, and K. Mullen, Chem.-Eur. J. 4, 2099 (1998).

${ }^{6}$ A. M. van de Craats, J. M. Warman, A. Fechtenkotter, J. D. Brand, M. A. Harbison, and K. Mullen, Adv. Mater. (Weinheim, Ger.) 11, 1469 (1999).

${ }^{7}$ L. Schmidt-Mende, A. Fechtenkotter, K. Mullen, E. Moons, R. H. Friend, and J. D. MacKenzie, Science 293, 1119 (2001).

${ }^{8}$ J. J. M. Halls, A. C. Arias, J. D. MacKenzie, W. S. Wu, M. Inbasekaran, E. P. Woo, and R. H. Friend, Adv. Mater. (Weinheim, Ger.) 12, 498 (2000).

${ }^{9}$ S. E. Shaheen, C. J. Brabec, N. S. Sariciftci, F. Padinger, T. Fromherz, and J. C. Hummelen, Appl. Phys. Lett. 78, 841 (2001).

${ }^{10}$ A. C. Arias, J. D. MacKenzie, R. Stevenson, J. J. M. Halls, M. Inbasekaran, E. P. Woo, D. Richards, and R. H. Friend, Macromolecules 34, 6005 (2001).

${ }^{11}$ M. G. Debije, J. Piris, M. P. de Haas, J. M. Warman, Z. Tomovic, C. D. Simpson, M. D. Watson, and K. Mullen, J. Am. Chem. Soc. 126, 4641 (2004).

${ }^{12}$ R. Iftimie, P. Minary, and M. E. Tuckerman, Proc. Natl. Acad. Sci. U.S.A. 102, 6654 (2005).

${ }^{13}$ O. A. von Lilienfeld, R. Lins, and U. Rothlisberger, Phys. Rev. Lett. 95, 153002 (2005).

${ }^{14}$ A. Maliniak, J. Chem. Phys. 96, 2306 (1992).

${ }^{15}$ I. Ono and S. Kondo, Bull. Chem. Soc. Jpn. 65, 1057 (1992).

${ }^{16}$ F. M. Mulder, J. Stride, S. J. Picken, P. H. J. Kouwer, M. P. de Haas, L. D. A. Siebbeles, and G. J. Kearley, J. Am. Chem. Soc. 125, 3860 (2003).

${ }^{17}$ G. Cinacchi, R. Colle, and A. Tani, J. Phys. Chem. B 108, 7969 (2004).

${ }^{18}$ J. A. C. Veerman and D. Frenkel, Phys. Rev. A 45, 5632 (1992).

${ }^{19}$ A. P. J. Emerson, G. R. Luckhurst, and S. G. Whatling, Mol. Phys. 82, 113 (1994).

${ }^{20}$ M. A. Bates and G. R. Luckhurst, J. Chem. Phys. 104, 6696 (1996).

${ }^{21}$ H. Zewdie, Phys. Rev. E 57, 1793 (1998).

${ }^{22}$ G. Cinacchi and A. Tani, J. Chem. Phys. 117, 11388 (2002). 
${ }^{23}$ D. Caprion, L. Bellier-Castella, and J. P. Ryckaert, Phys. Rev. E 67, 41703 (2003).

${ }^{24}$ L. Bellier-Castella, D. Caprion, and J. P. Ryckaert, J. Chem. Phys. 121, 4874 (2004).

${ }^{25}$ J. Baschnagel, K. Binder, P. Doruker et al., Adv. Polym. Sci. 152, 41 (2000).

${ }^{26}$ C. F. Abrams, L. Delle Site, and K. Kremer, Phys. Rev. E 67, 021807 (2003).

${ }^{27}$ L. Delle Site, C. F. Abrams, A. Alavi, and K. Kremer, Phys. Rev. Lett. 89, 156103 (2002).

${ }^{28}$ C. F. Abrams and K. Kremer, Macromolecules 36, 260 (2003).

${ }^{29}$ X. Zhou, D. Andrienko, L. Delle Site, and K. Kremer, Europhys. Lett. 70, 264 (2005).

${ }^{30}$ X. Zhou, D. Andrienko, L. Delle Site, and K. Kremer, J. Chem. Phys. 123, 104904 (2005).

${ }^{31}$ D. Andrienko, S. Leon, L. D. Site, and K. Kremer, Macromolecules 38, 5810 (2005)

${ }^{32}$ D. P. Craig and T. Thirunamachandran, Molecular Quantum Electrodynamics (Dover, Mineola, New York, 1998).

${ }^{33}$ S. Tsuzuki, T. Uchimaru, K. Sugawara, and M. Mikami, J. Chem. Phys. 117, 11216 (2002).

${ }^{34}$ W. Koch and M. C. Holthausen, A Chemist's Guide to Density Functional Theory (Wiley-VCH, New York, 2002).

${ }^{35}$ S. Kristyán and P. Pulay, Chem. Phys. Lett. 229, 175 (1994).

${ }^{36}$ J. M. Pérez-Jordá and A. D. Becke, Chem. Phys. Lett. 233, 134 (1995).

${ }^{37}$ E. J. Meijer and M. Sprik, J. Chem. Phys. 105, 8684 (1996).

${ }^{38}$ T. van Mourik and R. J. Gdanitz, J. Chem. Phys. 116, 9620 (2002).

${ }^{39}$ X. Wu, M. C. Vargas, S. Nayak, V. Lotrich, and G. Scoles, J. Chem. Phys. 115, 8748 (2001)

${ }^{40}$ T. A. Wesolowski, P. Y. Morgantini, and J. Weber, J. Chem. Phys. 116, 6411 (2002)

${ }^{41}$ T. A. Wesolowski and F. Tran, J. Chem. Phys. 118, 2072 (2003).

${ }^{42}$ Y. Andersson, D. C. Langreth, and B. I. Lundqvist, Phys. Rev. Lett. 76, 102 (1996).

${ }^{43}$ E. Hult, Y. Andersson, and B. I. Lundqvist, Phys. Rev. Lett. 77, 2029 (1996).

${ }^{44}$ H. Rydberg, B. I. Lundqvist, D. C. Langreth, and M. Dion, Phys. Rev. B 62, 6997 (2000).

${ }^{45}$ H. Rydberg, M. Dion, N. Jacobsen, E. Schröder, P. Hyldgaard, S. I. Simak, D. C. Langreth, and B. I. Lundqvist, Phys. Rev. Lett. 91, 126402 (2003).

${ }^{46}$ M. Dion, H. Rydberg, E. Schröder, D. C. Langreth, and B. I. Lundqvist, Phys. Rev. Lett. 92, 246401 (2004).

${ }^{47}$ A. J. Misquitta, B. Jeziorski, and K. Szalewicz, Phys. Rev. Lett. 91, 33201 (2003).

${ }^{48}$ W. Kohn, Y. Meir, and D. E. Makarov, Phys. Rev. Lett. 80, 4153 (1998).

${ }^{49}$ R. LeSar, J. Phys. Chem. 88, 4272 (1984).
${ }^{50}$ S. Grimme, J. Comput. Chem. 25, 1463 (2004).

${ }^{51}$ S. J. A. van Gisbergen, J. G. Snijders, and E. J. Baerends, J. Chem. Phys. 103, 9347 (1995)

${ }^{52}$ E. R. Johnson and A. D. Becke, J. Chem. Phys. 123, 24101 (2005).

${ }^{53}$ F. Ortmann, W. G. Schmidt, and F. Bechstedt, Phys. Rev. Lett. 95, 186101 (2005).

${ }^{54}$ O. A. von Lilienfeld, I. Tavernelli, U. Rothlisberger, and D. Sebastiani, Phys. Rev. Lett. 93, 153004 (2004).

${ }^{55}$ O. A. von Lilienfeld, I. Tavernelli, U. Rothlisberger, and D. Sebastiani, J. Chem. Phys. 122, 14113 (2005).

${ }^{56}$ O. A. von Lilienfeld, I. Tavernelli, U. Rothlisberger, and D. Sebastiani, Phys. Rev. B 71, 195119 (2005).

${ }^{57}$ E. Tapavicza, O. A. von Lilienfeld, I. Lin, M. Coutinho, I. Tavernelli, and U. Rothlisberger (unpublished).

${ }^{58}$ M. Coutinho, I. Lin, O. A. von Lilienfeld, I. Tavernelli, and U. Rothlisberger (unpublished).

${ }^{59}$ J. Hutter, P. Ballone, M. Bernasconi et al., Computer code CPMD, version 3.92, Copyright IBM Corp., 1990-2001, Copyright MPI-FKF, Stuttgart, 1997-2004; http://www.cpmd.org

${ }^{60}$ A. D. Becke, Phys. Rev. A 38, 3098 (1988)

${ }^{61}$ R. Colle and D. Salvetti, Theor. Chim. Acta 37, 329 (1975).

${ }^{62}$ C. Lee, W. Yang, and R. G. Parr, Phys. Rev. B 37, 785 (1988).

${ }^{63}$ J. P. Perdew, Phys. Rev. B 33, 8822 (1986).

${ }^{64}$ J. P. Perdew, K. Burke, and M. Ernzerhof, Phys. Rev. Lett. 77, 3865 (1996).

${ }^{65}$ J. P. Perdew and A. Zunger, Phys. Rev. B 23, 5048 (1981).

${ }^{66}$ D. M. Ceperley and B. J. Alder, Phys. Rev. Lett. 45, 566 (1980).

${ }^{67}$ S. Goedecker, M. Teter, and J. Hutter, Phys. Rev. B 54, 1703 (1996).

${ }^{68}$ C. Hartwigsen, S. Goedecker, and J. Hutter, Phys. Rev. B 58, 3641 (1998).

${ }^{69}$ M. Krack, Theor. Chim. Acta 114, 145 (2005).

${ }^{70}$ G. Martyna and M. Tuckerman, J. Chem. Phys. 110, 2810 (1999).

${ }^{71}$ R. Zacharia, H. Ulbricht, and T. Hertel, Phys. Rev. B 69, 155406 (2004).

${ }^{72}$ R. O. Contreras-Camacho, P. Ungerer, A. Boutin, and A. D. Mackie, J. Phys. Chem. B 108, 14109 (2004).

${ }^{73}$ M. D. Watson, A. Fechtenkotter, and K. Mullen, Chem. Rev. (Washington, D.C.) 101, 1267 (2001)

${ }^{74}$ C. D. Wick, M. G. Martin, and J. I. Siepmann, J. Phys. Chem. B 104, 8008 (2000)

${ }^{75}$ J. R. Errington and A. Z. Panagiotopoulos, J. Phys. Chem. B 103, 6314 (1999).

${ }^{76}$ P. Linse, J. Am. Chem. Soc. 106, 5425 (1984).

${ }^{77}$ P. Linse, G. Karlstrom, and B. Jonsson, J. Am. Chem. Soc. 106, 4096 (1984).

${ }^{78}$ M. Claessens, M. Ferrario, and J. P. Ryckaert, Mol. Phys. 50, 217 (1983).

${ }^{79}$ D. J. Evans and R. O. Watts, Mol. Phys. 32, 93 (1976). 